\title{
FUNCIONES DISCURSIVAS DEL LATÍN: CODE- SWITCHING EN TEXTOS DE MEDICINA ESPAÑOLES
}

Gabriela Cruz Volio

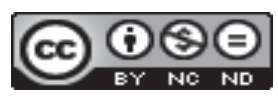

Esta obra está bajo una licencia Creative Commons

Reconocimiento-No Comercial-Sin Obra Derivada 



\title{
FUNCIONES DISCURSIVAS DEL LATÍN: CODE- SWITCHING EN TEXTOS DE MEDICINA ESPAÑOLES
}

\author{
LATIN LANGUAGE AND ITS DISCURSIVE FUNCTIONS: CODE- \\ SWITCHING IN SPANISH MEDICAL TEXTS
}

\section{Gabriela Cruz, Volio}

\begin{abstract}
RESUMEN
En este artículo se exponen los rasgos del code-switching del latín/español en textos de medicina españoles de la Edad Media y de la España renacentista y se exploran los usos de esta mezcla. En primer lugar se revisan los fundamentos para estudiar el code-switching en textos escritos y se alude a la historia de la escritura de textos de medicina en España en lengua vernácula, cuya elección lingüística está determinada por el público al que están dirigidos. En segundo lugar se describen las estructuras del code-switching y se examinan las funciones discursivas del empleo del latín en textos de medicina escritos principalmente en castellano. Se determina que la alternancia con el latín tiene que ver con a) cuestiones terminológicas, b) citación e intertextualidad y c) organización textual del manuscrito. Más allá de esto, la presencia del latín sirve como una práctica de escritura mediante la cual los autores orientan a los destinatarios y se posicionan a sí mismos. A través de las citas, el autor presenta una imagen positiva de médico instruido y construye un juego entre distintas voces que le sirven para exponer sus argumentos y de esta manera validarlos.
\end{abstract}

Palabras clave: code-switching, discurso médico, intertextualidad, terminología, vernacularización.

\begin{abstract}
This article examines the main features of Latin/Spanish code-switching in Medieval and Renaissance Spanish medical texts and its functions. The first sections discuss the theoretical implications of code-switching, specifically when studying written texts, and the history of Spanish medical writing. The following sections describe the code-switching structures and the discursive functions of this practice. It is argued that Latin code-switching relates to: a) terminological issues, b) citation and intertextuality and c) manuscript organization. Additionally, it is claimed that the Latin elements function as a writing practice through which the authors guide their readers and also position themselves. With the use of Latin citations, the author conveys a positive image of himself as a learned person and manages to display different voices which help support and validate his own arguments.
\end{abstract}

Key words: code-switching, medical discourse, intertextuality, terminology, vernacularization.

Dra. Gabriela Cruz Volio. Profesora invitada de la Escuela de Filología, Universidad de Costa Rica. Correo electrónico: gabriela.cruzvolio@ucr.ac.cr 


\section{El code-switching en textos escritos}

El code-switching, también llamado alternancia o cambio de código en español, se entiende como la yuxtaposición de fragmentos de lengua pertenecientes a dos sistemas o subsistemas linguiísticos distintos en un mismo evento comunicativo, según Gumperz (1977, p. 1). De acuerdo con Auer (1988), este fenómeno propio del habla bilingüe llamó la atención de un pequeño número de especialistas durante las décadas cincuenta y sesenta. Sin embargo, no es sino hasta los años setenta cuando surgen las primeras publicaciones sobre cuestiones sintácticas y sociolingüísticas del bilingüismo, ${ }^{1}$ lo cual da pie a que el code-switching se estudie desde la sintaxis, la sociolingüística y la psicolingüística, y que en las décadas posteriores la investigación sobre la alternancia de código se posicione como un tema relevante dentro de varias áreas de la lingüística. En este marco, se ha determinado que el code-switching no es simplemente la combinación de dos conjuntos distintos de reglas gramaticales, sino que integra dos sistemas lingüísticos (Wei, 2000, p. 14), por lo que se ha investigado sobre los aspectos morfosintácticos que caracterizan a este fenómeno. Más allá de eso, se ha insistido en que el code-switching transmite y crea significado social y comunicativo (Auer, 1988, pp. 1-2), razón por la cual el análisis del cambio de código requiere tomar en cuenta el contexto sociocultural y conversacional en el que se da.

A pesar de la evolución en este campo del bilingüismo, el code-switching se ha abordado mayoritariamente a través del lenguaje hablado, tanto en situaciones informales como institucionales, mientras que todavía hace falta explorar el empleo del code-switching en la gran diversidad de textos escritos, desde poemas medievales hasta correos electrónicos, que presentan esta práctica comunicativa (Sebba, 2012). Esto no quiere decir que no existan trabajos sobre el code-switching en el medio escrito. De hecho, se cuenta con investigaciones desde inicios de los sesentas, ${ }^{2}$ cuyo interés principal ha girado alrededor de las representaciones dialógicas en textos escritos. En estos primeros estudios, las fuentes constituían un medio para tener acceso a prácticas conversacionales, pero se consideraban como secundarias con respecto a la oralidad propiamente. Si bien ha habido un desarrollo significativo en la concepción del code-switching en el medio escrito, en donde se ha pasado de la descripción de las restricciones sintácticas de este fenómeno a la consideración del texto como un elemento autónomo dentro de una cultura multilingüe que permite ahondar sobre las funciones tanto locales como globales del code-switching, ${ }^{3}$ se necesitan no solo más estudios empíricos sino sobre todo un marco teórico independiente para comprender la alternancia de códigos en textos escritos.

Al acercarse al cambio de código desde una perspectiva histórica, este es un punto especialmente relevante, ya que solo se cuenta con testimonios escritos. Por otro lado, los trabajos que se han realizado recientemente sobre el code-switching en documentos legales de inglés medieval tardío (Davidson, 2005), en textos de remedios del siglo XIII escritos principalmente en occitano (Niiranen, 2011), en textos médicos del inglés moderno temprano (Pahta, 2011), en la correspondencia privada entre mujeres inglesas durante 1400 y 1800 (Nurmi y Pahta, 2012), en las jarchas arábico-romances durante los siglos XI y XIV (Thomas y Sayahi, 2012), así como en diversos documentos anglosajones y sermones medievales (Schendl, 2012), por citar algunos ejemplos, ${ }^{4}$ evidencian la amplia variedad de textos en los que se puede estudiar el code-switching y la viabilidad de emprender investigaciones diacrónicas sobre este fenómeno. En el caso particular de la historia del español, todavía hay una carencia de estudios que se encarguen de las prácticas del code-switching y el bilingüismo en textos escritos. ${ }^{5}$ 
Con el fin de contribuir a esta área de conocimiento, este trabajo se concentra en las formas y las funciones discursivas del code-switching latín/español en textos de medicina producidos entre los siglos XIII y XVII. Por la naturaleza de los documentos, concebidos en un medio escrito, se parte del marco teórico de Sebba sobre el code-switching en esta modalidad:

\begin{abstract}
I will argue here for a new approach to written mixed-language discourse, with three key points: (1) the study of written mixed-language discourse needs to be situated within a broader field which deals with the semiotics of mixed-language texts in the broadest sense; (2) the production and reading of mixed-language written texts needs to be studied within a literacy framework, in order to understand the acts of writing, reading and language mixing within the context of literacy practices of which they are a part; (3) visual and spatial elements of the written form potentially provide important contextualisation cues (in other words, are an integral part of the interpretation of the message) and therefore need to be included in any framework which seeks to do justice to the semiotics of written mixed language texts, even though they may not be relevant to all such texts. (2012, p. 3)
\end{abstract}

Aparte de la consideración de los textos escritos en su complejidad semiótica, es muy importante destacar el segundo punto sobre la producción y recepción de textos caracterizados por la mezcla de idiomas en el marco de la lectoescritura. Así, la escritura y la lectura de textos se concibe como una práctica social que forma parte de un contexto histórico (Sebba, 2012, p. 8). Por lo tanto, la propuesta de Sebba (2012, p. 17) empieza por comprender las condiciones de las prácticas de composición y de lectura para lograr así interpretar las funciones del codeswitching en un texto particular.

\title{
2. Textos de medicina medievales y renacentistas en lengua vernácula: Un nuevo público
}

Si bien entre los textos de carácter científico elaborados en el taller del rey Alfonso el Sabio a mediados del siglo XIII no aparece un tratado de medicina específico, en sus obras surge una cantidad importante de voces que atañen a la medicina, como enfermedades, lo cual se relaciona directamente con el avance de esta ciencia entre 1250 y 1350, de acuerdo con Clavería Nadal (2004, p. 73). ${ }^{6}$ A pesar de esta nada despreciable producción alfonsí, cuando el número de textos médicos en castellano aumenta es a finales de la Edad Media y en el siglo XVI con los procesos de vernacularización. ${ }^{7}$ De acuerdo con Gutiérrez Rodilla (1998, p. 64), este giro hacia la escritura en lengua romance está conectado con el valor de herramienta política que han ido adquiriendo las lenguas vernáculas hacia el siglo $\mathrm{XVI}^{8}$ y su rol en las unificaciones nacionales, lo cual se plasma también en la creación de los primeros diccionarios escritos exclusivamente en romance.

Por otro lado, la gran cantidad de traducciones de textos de carácter científico que se había realizado desde el siglo XIII a partir del latín, en mayor medida, y la redacción de textos médicos en lenguas vernáculas suponen la divulgación de los saberes a un público más amplio. ${ }^{9}$ En este contexto, las obras de materia medica compuestas en lenguas romances toma fuerza en los siglos XV y XVI. Según Gutiérrez Rodilla (2009, pp. 38-39), gran parte de estas nuevas ediciones vernáculas estarían dirigidas a médicos que no habían recibido una educación universitaria y que no tenían conocimientos de latín. Dentro de este grupo mundo de médicos medievales, se encontraban los cirujanos-barberos, los herboristas y los boticarios, ${ }^{10}$ por ejemplo, encargados de realizar tareas consideradas menores. Consecuentemente, "[...] la utilización del vulgar en los textos médicos estuvo estrechamente relacionada con el contenido de la obra y su destinatario y fue el resultado de una necesidad impuesta por la realidad" (ibid., p. 40). Debido a estos factores, las obras médicas en castellano se dividen en dos 
grandes grupos, de acuerdo con Gutiérrez Rodilla (ibid.): a) obras instructivas, compuestas para dirigir la práctica de cirujanos, sangradores, comadres y boticarios, entre otros, y b) obras de terapéutica y regímenes sanitarios, escritas para la curación personal de quienes no tenían acceso a los servicios médicos y en las que se trataba ya fuera de contenidos novedosos, quizás controversiales, o de recomendaciones preventivas en épocas críticas de salud.

Ahora bien, ya fueran textos dirigidos a un público sin conocimientos de la lengua latina, y a pesar de que las lenguas vernáculas habían ido ganando terreno, lo cierto es que la elección de una lengua u otra no era un asunto neutral. Así, de acuerdo con Santamaría Hernández (1999, p. 120), en el siglo XVI los profesionales valencianos discuten sobre las consecuencias de escribir en castellano. ${ }^{11}$ Por una parte, hay quienes opinan que con la lengua romance se puede alcanzar a un público más amplio, entre el cual se encuentran los cirujanos y otros practicantes sin formación universitaria. Por el contrario, algunos consideran que escribir sobre medicina en lengua vulgar atenta contra los principios humanísticos que solo se pueden lograr mediante el latín y la imitación de las autoridades. Consecuentemente, la elección de la lengua latina se asocia con la "elite científica del momento y de sus representantes más importantes en nuestro país y en el extranjero" (Santamaría Hernández, 2003, p. 733), mientras que la escritura en lengua vernácula podía dañar el prestigio de los profesionales, de acuerdo con Gutiérrez Rodilla (2009, p. 38), no solo por la escogencia lingüística, sino por la materia tratada (temas prácticos y específicos) y por el tipo de público al que estaban dirigidos los textos en romance.

\section{Sobre el corpus y la selección de pasajes de code-switching}

Como material se ha recurrido al corpus digital de textos médicos españoles de la Digital Library of Old Spanish Texts elaborado por el Seminario de Estudios Medievales Hispánicos (HSMS, por sus siglas en inglés) de la Universidad de Wisconsin en Madison. Este corpus abarca un total de 58 textos de medicina que van desde el siglo XIII hasta el siglo XVII. Los textos se caracterizan por su amplia variedad, pues se encuentran tratados generales y específicos sobre enfermedades particulares como la sífilis, asuntos ginecológicos y métodos de diagnóstico, así como recopilaciones de remedios y recetas, guías de salud y tratados quirúrgicos y anatómicos (Gago Jover, Herrera y González de Fauve, 2015). Aparte del material textual, el sitio cuenta con índices alfabéticos y las listas de concordancias de cada texto. ${ }^{12}$

El primer paso de este trabajo ha consistido en la ubicación de pasajes en latín dentro de los textos. Estos segmentos latinos, que van desde grupos nominales y frases preposicionales hasta complejos clausulares, forman parte de lo que se considera code-switching en el presente estudio: el uso de más de una lengua en un solo episodio comunicativo (Heller, 1988, p. 1). La amplitud de esta definición, que engloba toda mezcla de idiomas que sea identificable, es ideal para un acercamiento cuyo punto de partida sean los textos mismos (Pahta, 2011, p. 118). Ahora bien, esta identificación de elementos supone el problema teórico de ver si se trata efectivamente de cambios de código o de préstamos léxicos, ${ }^{13}$ por lo cual se sigue la solución de Pahta (2011, pp. 120-121) de considerar los elementos sin acomodación morfológica al español, así como las expresiones que el autor marca de manera explícita como no castellanas. Además, de acuerdo con Crespo y Moskowich, se considera que el code-switching es utilizado conscientemente por los hablantes, en oposición al empleo de préstamos, y que es una estrategia que sirve para marcar la pertenencia de grupo: 
We contend that code-switching is a communicative resource which authors/speakers use in a conscious way whereas borrowing, precisely because it is more integrated in the language, is unconscious. Likewise, we agree with Scotton's conception $(1983,1988)$ of code-switching as a socially marked, in-group strategy (Crespo y Moskowich, 2006, pp. 52-53)

En cuanto al análisis, la atención no ha recaído sobre las limitaciones morfosintácticas del code-switching, sino sobre las funciones discursivas que conlleva esta práctica comunicativa en textos de medicina de la época. Por consiguiente, la interpretación de los datos se presenta mediante una perspectiva cualitativa para tomar en cuenta la información cotextual y contextual del empleo de elementos latinos en textos escritos principalmente en castellano.

\section{Estructuras y funciones del code-switching en los textos médicos}

En las siguientes subsecciones se presenta el análisis de los datos. Para este propósito, primero se describe la estructura sintáctica de los fragmentos latinos que se insertan en los textos de medicina. Seguidamente se examinan las funciones discursivas y textuales que cumple el uso de elementos y pasajes en latín.

Con respecto a los textos estudiados, se ha observado que la presencia de elementos latinos se da en todas las épocas, aunque en los textos más tempranos, como los Lapidarios del siglo XIII, el code-switching se emplea sobre todo para referirse a asuntos terminológicos, mientras que los fragmentos latinos para citar autoridades clásicas es propia de textos posteriores. Por otro lado, si bien la mayoría de los pasajes en lengua latina se encuentran en el cuerpo del texto, en algunas obras, como el Discurso y despertador preservativo, el escritor a veces incluye la cita en el margen del manuscrito como parte de la marginalia.

\subsection{Caracterización sintáctica del code-switching}

Los fragmentos latinos que se utilizan en los textos de medicina estudiados van desde elementos nominales individuales, grupos nominales y frases preposicionales hasta cláusulas, complejos clausulares y secuencias textuales. Los elementos y grupos nominales por lo general hacen referencia a nombres de hierbas, plantas medicinales o enfermedades:

1. Yo me hallaua muy bien \& hazia yo muy marauillosa obra con la magna y quando no la tenia vsaua de la magna de canaria, la qual en esta primera especie faze marauillosa obra aparejando con jarope de fumus terra \& vna onça de magna con su cañasistola disoluida en fuero de leche de cabras [...]. (Contra el mal serpentino 1542, fol. 7v)

2. Por muchas causas se suelen detener las pares que en latin se dizen Secundinae, las quales comunmente salen con la criatura. (Libro del parto humano 1580, fol. 56v)

En el ejemplo 1, el autor describe una forma de curación y habla del jarabe de la hierba fumus terra. Aunque en este caso se deja el nombre en latín, el término correspondiente en castellano, 'fumaria', aparece en otros tratados médicos del siglo XVI. En el ejemplo 2, se utiliza el nombre del latín tardío secundinae a modo de explicación del término en español 'pares', utilizado en plural, que denomina al "órgano que envuelve el feto en el vientre de su madre y que se expulsa en el parto" (Lleal, 2017), es decir, la placenta.

Otro de tipo de palabras individuales que se emplean en latín tiene que ver con elementos de índole formulaica, como fórmulas de agradecimientos (gratias) y de saludos y despedidas (vale). Estas formas se utilizan ya sea en los prólogos o en las dedicatorias cuando se dirigen a la persona que había comisionado el tratado o bien en el cierre de la obra. 
Dentro del texto que abarca la materia medica propiamente, se insertan palabras latinas sueltas que cumplen una función metadiscursiva, como et cetera, iterum, item y la forma combinada item mas. ${ }^{14}$ De acuerdo con Hyland (2005, p. 50), estos recursos interactivos organizan la información de manera que esta sea presentada de manera accesible al lector. Además, su uso marca la recurrencia a una norma de escritura específica relacionada con el registro académico de estos textos. ${ }^{15}$ Lo mismo sucede con ciertas frases estandarizadas, como sine qua non, quid pro quo y extra principium, entre otras, que se introducen en el texto español:

3. Y sino es causa conjunta la caluicies, es causa necessaria, que dizen, Sine qua non, Que si es menester causa material, [...] (Discurso de la gota 1594, fol. 9r,v)

4. [...] y miren como tendran el Abrotano verdadero, pues les venden la mançanilla loca por el, y no reparan el gran daño que hazen en poner, quid pro quo, y lo peor de todo, ser vna yerva que no tiene virtud, alguna mas de la que se ha dicho en su capitulo. (Tratado de raras y peregrinas yervas 1674 , fol. 41r)

En el ejemplo 3, el escritor de la obra traduce la expresión sine qua non, "es causa necesaria", lo cual refleja la conciencia del público al que se está dirigiendo. En el prólogo, el médico Juan Cornejo dedica la obra al rey don Felipe Segundo y manifiesta que la ha escrito para que el rey ordene su lectura al pueblo, por lo cual el manejo del latín no es esperado: " $A$ Vuestra Magestad humilissimamente suplico se sirua de mandallos leer, y ver si dan gusto, y tienen facilidad, [...]" (Discurso de la gota 1594, fol. 5r).

Por otro lado, también ocurren otras frases de un carácter o bien menos fijo o no relacionado con la organización del contenido, como cum grano salis y pro manibus:

5. Esta auctoridad se ha de entender cum grano salis: [...]. (Libro de las comadres 1541, fol. 70r)

6. Viene el ydiota que lo trahen pro manibus y dan cuenta de todo lo passado \& dize que la fiebre \& las camaras \& la opilacion todos son los accidentes de la enfermedad del morbo serpentino que quanto los fisicos le han fecho ha sido dañalle [...].(Contra el mal serpentino 1542 , fol. 78 r)

Todos los ejemplos que se han presentado son ejemplos de code-switching intraoracional, en tanto que los elementos latinos se dan dentro de la misma oración o fragmento oracional del español (Myers-Scotton, 1993, p. 4). Además, la alternancia de código también se da al insertar cláusulas tanto subordinadas como independientes en latín dentro del texto. En ambos casos se trata de code-switching interoracional (ibid.,p. 3), como se observa en los dos ejemplos siguientes:

7. Y destos tales dezia en mi tiempo aquel buen doctor huius anima in pace requescat don ximen Perez de Figuerola Visorey de Mallorca. (Libro de las comadres 1541, fol. 30r)

8. E eso mismo de los dichos de Galieno en el regimiento de las agudas sobre aquella parte. Quando dolor ad spatulas. E esso mismo se prueua de los dichos de Galieno .xiij. tractado de ingenio capitulo .vj[.]. (Lilio de la medicina 1495, fol. 51r)

El ejemplo 7 incluye el uso de una cláusula de tipo relativo con el pronombre huius en vez de cuius. Al tratarse de un epitafio latino, por un lado participa de un carácter formulaico y, por otro, el cambio de código marca también el paso de un tipo de texto, el de medicina, a otro, el de la inscripción sepulcral. 
En el ejemplo 8 se intercala una cláusula temporal en latín. En este pasaje, el autor está hablando de Galeno y de hecho lo ha citado recientemente. Después, al continuar con el texto en español, deja la cláusula temporal en latín. En este caso el code-switching cumple una función referencial, ya que remite al lector a la parte del texto de Galeno ${ }^{16}$ que recomienda la flebotomía para curar la pleuresía cuando uno de los síntomas es el dolor de omóplatos. Estas inserciones reflejan el evidente contacto de los médicos españoles con textos en lengua latina.

Cuando aparece una sola cláusula en latín dentro del texto, es común que se trate de intertextualidad. En algunos casos, el escritor no cita al autor original ${ }^{17}$ y además se trata de fragmentos recurrentes en textos medicinales de la época y de Europa, no solo de España. Así, en el siguiente ejemplo se inserta un dicho atribuido a Aristóteles, sin que sea mencionado por el autor:

9. [...] \& si todos los lugares estuuieren corruptos mejor \& mas seguro es cada vno estar en su proprio lugar ((quia locus est conseruatiuus locati)) saluo si el tal lugar proprio estuuiesse en mal sitio [...]. (Tratado contra toda pestilencia 1518, fol. $3 r)^{18}$

Por otra parte, los pasajes en latín que abarcan un conjunto de oraciones surgen cuando el escritor del tratado cita explícitamente a otra autoridad:

10. lo mesmo dixo Hippocrates. Producuntur enim omnes sursum habentes caput pariuntur autem multi in caput \& securius procedunt \& liberantur his qui in pedes pariuntur, y en otra parte. Si foetus inclinatur per caput facilius parit si autem obliquus, \& in pedes procedit, \&caetera: [...] (Libro del parto humano 1580, fol. 16v, 17r)

En el ejemplo 10, se observa cómo el autor alterna las citas de Hipócrates con fragmentos textuales en español ( $y$ en otra parte).

Otros lugares de las obras que presentan pasajes extensos en latín son las recetas médicas, los prólogos y las dedicatorias. En el Libro de las comadres, la mayoría de las recetas están escritas en lengua latina, lo cual no sucede en el resto de las obras:

11. Recipe sirupi de plantagine dracmas .iij. aque violarie dracmas .iiij. misceantur \& in duabus vicibus reiteretur. Y denle este secreto. (Libro de las comadres 1541, fol. 94v)

En cuanto a los prólogos y las dedicatorias, el autor a veces incluye una versión completa en español que le sigue al texto latino. Esto sucede, por ejemplo, en Tratado contra la pestilencia $(1518$, fol. $1 \mathrm{v})$, Contra el mal serpentino $(1542$, fol. $1 \mathrm{v})$, Tractado del uso de las mugeres (1586, fol. 25v) y Libro de experimentos médicos $(1598$, fol. 1r). Esta forma de presentar un contenido similar pero en lenguas distintas es lo que Sebba (2012, p. 14) llama 'textos paralelos', pues se da una relación de paralelismo entre dos fragmentos textuales en una misma composición. En efecto, pareciera que los textos estaban dirigidos a públicos distintos: los miembros de la nobleza y la comunidad médica o el pueblo. ${ }^{19}$ A diferencia de lo que sucede en algunos prólogos, la alternancia con el latín dentro del cuerpo de la obra tiende a darse en una relación de complementariedad (ibid., p. 15), pues los pasajes en lengua latina presentan un contenido distinto del texto en español. Ahora, por la naturaleza de estos textos médicos, no hay que dejar de lado que muchas veces el autor parafrasea o explica el pasaje citado en latín. Además, a veces se refiere a términos de enfermedades o de plantas tanto con el término latino como el castellano, como se observó en el ejemplo 1. Estos dos últimos aspectos se revisan más adelante. 


\subsection{Funciones discursivas y textuales del code-switching}

Como se observó en la sección anterior, el uso del latín en los textos de medicina españoles de la Edad Media y del Renacimiento se da de varias formas que van desde palabras individuales hasta pasajes relativamente extensos. Ahora, el empleo de otro idioma distinto al castellano cumple diversas funciones, como nombrar enfermedades, hierbas y obras de medicina antiguas, validar lo dicho por el autor mediante citas, establecerse como un miembro de la comunidad científica y organizar el contenido textual del manuscrito. A continuación se elabora sobre las tres funciones principales que cumple el code-switching en este tipo de texto científico: empleo de terminología médica, referencias y argumentación y organización del texto. ${ }^{20}$

\subsubsection{Terminología médica}

Debido a que los textos de medicina en castellano de esta etapa se basan en obras de la antigüedad y que muchas de ellas son traducciones del latín, ${ }^{21}$ gran parte del uso de esta lengua tiene que ver con la designación de términos propios del registro médico. Consecuentemente, para nombrar enfermedades se recurre al latín:

12. [...] deuese temer muy difficil enfermeda en la parida, muy mayormente ahogamiento, que se dize, strangulatio vteri. (Libro del parto humano 1580, fol. 57r,v)

13. Desto quedo el Medico tan vfano, que començo ante otros Medicos a pregonar su medicamento, contra morbum incurabilem. (Manual contra la gota 1589, fol. 24v)

Igualmente, a veces se conserva el nombre original de instrumentos, medicamentos y plantas medicinales:

14. [...] y la atrayga, o con vn instrumento que llama Galeno. Pyulca .1. puris attractura. (Libro del parto humano 1580, fol. 106v)

15. [...] se pueda dezir desta, lo que dizen los Griegos de las mas raras, y singulares, Medicinas prouerbialmente. Panchrestum medicamentum, que significa medicina para toda cosa. (Manual contra la gota 1589, fol. 33r)

16. Çannahoria es dicha pestinaca en latyn \& çannaforia en rromançe Et esta yerua es de tal virtud que sy es bien cocha \& comjda \& el çumo della es beujdo aprouecha muncho al dolor del baço [...]. (Macer herbolario 1401-1500, fol. 7v)

17. [...] contra el escalentamjento del figado poluos con agua rrosada [fol. 21r] o con sugo de solatrum [...]. (De las melecinas 1401-1500, fol. 20v-21r)

Los ejemplos 14 y 15 muestran que el latín no es la única lengua del code-switching en estos textos médicos, pues aparecen también términos en griego. ${ }^{22}$ En el ejemplo 14, el

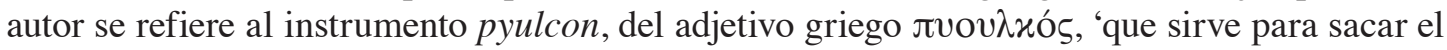
pus', ${ }^{23}$ cuya invención se atribuye a Galeno. En principio esta herramienta se usaba para extraer sustancias del tórax, lo cual se especifica mediante el latín puris attractura, 'atracción del pus', pero el autor del tratado en este caso lo recomienda para extraer el feto muerto de la madre. En el ejemplo 15, se habla de la medicina multiuso panchrestum medicamentum, conformada por

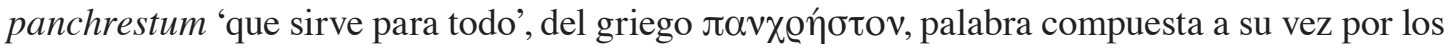

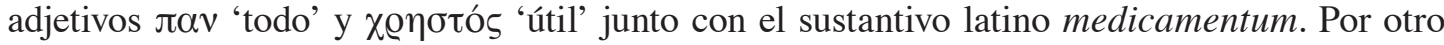
lado, los ejemplos 16 y 17 presentan nombres de plantas medicinales en latín: pestinaca, cuya 
forma latina correcta es pastinaca, y solatrum, término del latín tardío para la planta solanum nigrum 'hierba mora', ${ }^{24}$ una especie de planta venenosa.

El uso de terminología latina sirve para inscribir los textos dentro del dominio de conocimiento particular de la medicina. En este sentido, señala la pertenencia de grupo de la comunidad científica, dentro de la cual se encuentran profesionales en su mayoría familiarizados con el latín. ${ }^{25}$ Ahora, es usual que el término latino sea confrontado con su equivalente en romance o castellano y que se aclare el porqué de los nombres:

18. Et a tal uertud que tuelle la dolor que se faze en la media cabeça aque llaman en arauigo xaqueca. \& en latin migranea. (Lapidarios 1200-1300, fol. 8r)

19. Ay otra fleuma que en latin es llamada vitrea \& por esta rrazon le pusyeron atal nonbre porque en color \& en sabor semeja vidrio (Tratado de las fiebres 1450-1500, fol. 122v)

20. Ca la que passa allende en grossura. en griego llamase herpestiomeno. y en latin cancer. (Tratado de cirugía 1498, fol. 34v)

21. [...] y dormecella por razon de aquel calor y virtud que tiene que haze el neruezillo de la dentadura insentible, como se puede ver por la ethimologia desta yerua que llaman en castellano pelitre, y en latin piretrum y en griego, piretron que viene y se deriua a pir, que quiere dezir fuego. (Tratado de la boca y dentadura 1570, fol. 75v)

En los Lapidarios del rey Alfonso $\mathrm{X}$ el interés recae principalmente sobre las aclaraciones de vocablos latinos, griegos y árabes. En el ejemplo 18 el autor alude al término latino migranea, que proviene de la variante del latín tardío hemicrania (y este a su vez del

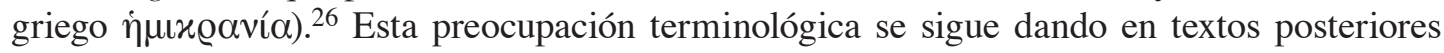
mediante la comparación linguiística y comentarios etimológicos. Así, en 19, se aclara la razón del uso de la palabra latina vitrea, del adjetivo vitreus, 'de vidrio'. En el ejemplo 20, se confrontan dos términos de otras lenguas. Esta vez el escritor del tratado se refiere a la enfermedad 'herpe estiómeno', ${ }^{27}$ la cual según él equivale al latín cancer, que quiere decir 'tumor maligno ulceroso'. ${ }^{28}$ En el ejemplo 21 se explica la etimología del término castellano pelitre ${ }^{29}$ que deriva del latín piretrum (pyrethrum) y este del griego piretron ( $\pi u ́ \varrho \varepsilon \theta \varrho o v$ ).

Por una parte, las definiciones y aclaraciones de vocabulario obedecen a la necesidad de crear un lenguaje de la medicina en español, lo cual forma parte del proceso de vernacularización ${ }^{30}$ de estas obras. Por otro lado, este fenómeno también se debe a que muchos de estos textos fueron escritos para un público no profesional o que no tenía conocimientos de latín, aunque formaran parte del mundo de la medicina, como se indicó anteriormente. Por consiguiente, los comentarios terminológicos reflejan la conciencia de los escritores con respecto a sus lectores.

\subsubsection{Referencias y argumentación}

La mención de obras médicas antiguas en latín ${ }^{31}$ es una práctica de intertextualidad mediante la cual los autores se refieren a un libro conocido, como se observa en los siguientes ejemplos. En ocasiones se indica también el nombre o el número del capítulo relevante en latín:

22. E auiçena enel su libro de regimine sanitatis. Dize que el buen manjar: cria buena sangre. (Macer 1518, fol. 1v) 
23. y esto dize el Galeno enel .xx. de vtilitate particularum capite .v. como dize. Cum infans natus est non plus deppendet a fecundina nec per vmbilicum: sed per os alitur depercollum vecisse vrinain emittit euident rescedunt tres vmblici vtilitates. $Y$ por esso se ata: porque no salga el sangre y se corta. (Libro de las comadres 1541, fol. 47v)

Por lo general, el escritor indica la fuente a la que se refiere dentro del cuerpo del texto, pero en obras como el Libro del parto humano y el Tractado del uso de las mugeres, el título de la obra aludida se escribe al margen del manuscrito. Esta forma de organización textual se revisa más adelante (sección 4.2.3).

Puesto que se trata de casos de intertextualidad, la mención de otro texto médico suele servir para introducir una cita directa del texto latino, lo cual se observa en el ejemplo 24, más abajo. Por un lado, referirse a otras autoridades médicas indica que los escritores asumen que comparten este conocimiento con el público al que se dirigen. En este sentido, las citas son un recurso metadiscursivo, de acuerdo con Hyland (2005, pp. 49-51). Por otro lado, aparte de enmarcar el trasfondo de la tradición erudita de la medicina al aludir a la fuente de conocimiento, citar directamente en latín es un mecanismo mediante el cual los autores construyen su identidad de expertos que además pertenecen a la élite que domina esta lengua, ${ }^{32}$ como señala Pahta (2011, p. 125). Asimismo, Gutiérrez Rodilla insiste en que la función de la cita de autoridades radica en otorgarle a los escritores credibilidad:

[...] ante sus propios colegas, quienes seguro no verían con buenos ojos tanto empeño en divulgar medicina: de este modo les mostraba que ese empeño no estaba reñido con tener un excelente conocimiento de las grandes autoridades médicas, así como una formación tan sólida como la de cualquiera de ellos. (2009, p. 44)

Por eso no es de extrañar que los escritores suelan traducir o parafrasear las citas, pues la importancia de los segmentos en lengua latina es su contraposición lingüística con el texto castellano: ${ }^{33}$

24. No dexo con todo esto dios de dar les ciencia para guardar se delas cosas a ellos dañosas/ y proueer enlas cosas necessarias como dize Tulio enel primero de sus oficios capitulo quarto/ diziendo. Homo autem qui rationis est particeps. \&cetera. Que quiere dezir: que dios poderoso dio capacidad al hombre y entendimiento para usar de razon: por la qual es muy differente delos otros animales. (Libro de las comadres 1541, fol. 6r)

25. Y por esso dize la auctoridad del Philosopho. Sol \& homo generant hominem que quiere dezir. El sol y el hombre engendran el hombre. (Libro de las comadres 1541, fol. 10v)

26. SI por ninguna cosa se ha de prohibir el vino a los deste mal, es por lo que dize San Pablo a los Ephefios Nolite inebriari vino, in quo est luxuria que significa, no beuays vino demasiado, porque es el promouedor de la luxuria. (Manual contra la gota 1589, fol. 46v)

27. Por esso dixo bien la otra regla medicinal y prouerbial Dum caput dolet cetera membra dolent que doliendo la cabeça no pueden dexar de doler, o enfermar los otros miembros del cuerpo (Manual contra la gota 1589, fol. 76v)

28. [...] como lo afirma Auicena sentencia .I. tertij, tractatu .4 capitulo .I. diziendo: Multa mota nocent, quae si non mouerentur, non nocerent, que muchas cosas por mouerlas, dañan, que si no se mouiessen, no dañarian. (Defensa de las criaturas de tierna edad 1604, fol. 30r) 
29. El castoreo siendo rancioso, negro, y hediendo, puede matar a vn enfermo en vn dia natural: Cabeat Medicus, \& Apotecarius a Castoreo nigro, \& rancido, nam tale interficit die vno. (Tratado de raras y peregrinas yervas 1674, fol. 15r)

Además, al citar en latín se realizan distintas funciones argumentativas. Según Hyland (2005, p. 51), el uso de citas influye en la interpretación que los lectores hacen del texto y sirve para dejar claro el dominio de las autoridades relacionadas con el tema manejado. Por ejemplo, a veces las citas se usan para referir al lector a la obra en la cual se está basando, por lo cual no aparecen completas. Esto sucede a lo largo del texto Lilio de la medicina ${ }^{34}$ en donde los pasajes latinos suelen cerrarse con la expresión \& cetera:

30. Estas condiciones se leen de Galieno en el regimiento de las agudas sobre aquella parte. His autem qui pleuretici sunt. \& cetera. (Lilio de la medicina 1495, fol. 7v)

31. E aquesto dize Galieno en el octauo de ingenio \& en el libro de complesion donde dize que la costumbre es otra natura \& esto mismo dize ypocras quando dize. Parum deterior cibus et potus \& cetera. E quando dize ex multo tempore asueta \& cetera. E quando dezia. asueti asuetos ferre labores \& cetera. E quando dezia mala dieta cibus \& potus \& cetera. (Lilio de la medicina 1495, fol. 128v)

Sin lugar a dudas, la función retórica más prominente de las citas en latín consiste en reforzar lo dicho por el autor. Por ejemplo, las obras de medicina escritas en castellano durante esta época eran por lo general textos instructivos, como se observó en el apartado 2, manuales prácticos cuya finalidad consistía en que el destinatario cumpliera con las instrucciones del médico escritor. Así, para resaltar la eficacia de una indicación, los escritores citan en latín a una autoridad médica de la tradición erudita que respalde lo aconsejado por ellos. Esto se observa en el Libro de las comadres, manual de instrucciones supuestamente dirigido a las parteras:

32. [...] como dize Auicenna la causa. Si est de illis que declinare faciunt ad matricem materiam: precipue calidad: ex qua timetur apostema: curetur cum eo quod de solutione est possibile. Hauemos concluydo que conuiene euacuacion con farmacia: y esto de mucha necessidad: pues en otra manera seria peligro de mal parrir/ o de morir la criatura como dizel mesmo doctor mas abaxo. Ibi imo timetur vt consequatur embrionem causa eius nocumentum aut lesione faciens ipsum abortiri aut interficiens ipsum. (Libro de las comadres 1541, fol.22v)

33. Y muchas vezes viene el mal parir por mucha humidad retenida enla matriz/ la qual la haze lubricar: y por ende mal parir segun vemos por esperiencia y lo dize Auicenna enel lugar allegado/ diziendo. Propter lubricitatem vero ad humiditatibus \& cetera. Por la qual razon es menester proueer enello con sus melezinas desta manera. (Libro de las comadres 1541, fol. 23r)

34. Tambien el anis, el hinojo, verde y no seco, las almendras, las auellanas multiplican esperma, y el vino fuerte y dulce, en el qual esta la luxuria, como lo dize sant Pablo, y Aristoteles, en los Problemas, dixo.Vinum uenereos facit.Y Ouidio en el de remedio amoris. Vina parant animos Veneri. etcaetera (Tractado del uso de las mugeres 1586, fol. 14r)

En el ejemplo 32, el médico Damián Carbón, quien escribió el Libro de las comadres, aconseja que se utilice un preparado para provocar el parto. El tono de insistencia se marca por su comentario y esto de mucha necesidad y mediante las citas en latín, ya que el médico 
respalda su exhortación con lo recomendado por Avicena. En 33, el mismo autor explica una de las causas del aborto e incluye un extracto de la fuente de donde ha obtenido la información a modo de apoyo. Con base en este fundamento, el médico concluye que es menester darle los medicamentos a la mujer. En 34, el escritor del tratado sugiere el uso del vino como afrodisiaco, cuya eficacia, según él, se comprueba a través de los textos de San Pablo, Aristóteles y Ovidio.

Aparte de intentar convencer al destinatario de que siga una recomendación, el latín también se emplea para intensificar lo dicho por el autor. Esto se observa particularmente en el uso de refranes o frases cortas y puntuales en latín que aluden a juicios de valor considerados universales, por lo cual funcionan como una máxima moral ${ }^{35}$ que sirve para persuadir al destinatario de la veracidad y de la importancia de lo dicho por el escritor. Por su carácter universal, no se suele mencionar el origen de la cita: ${ }^{36}$

35. E dezimos que todas las grossuras hazen engrossar a quien las mucho vsa comer. Grossus cibus: grossum sensum generat. (Macer 1518, fol. 28r)

36. Seteno deuedes de notar que el coytu comiença del celebro por razon de la ymaginacion del qual procede \& se demedia por el figado por razon del calor: Acerca de aquello. Cogit amare iecur. E cumplese por razon de los testiculos: acerca de aquello. (Lilio de la medicina 1495, fol. 167r)

37. [...] porque siendo rico el pleueyo, parece noble, el ignorante, y necio, es sabio, porque todo obedece al dinero: omnia obediunt pecuniae. (Tratado de raras y peregrinas yervas 1674, fol. 13r,v)

Como se observa en los ejemplos 35,36 y 37, el texto latino está traducido o parafraseado en castellano. De acuerdo con Gumperz, esto se debe a que la finalidad del code-switching en estos casos es enfatizar un enunciado: "Frequently a message is repeated in the other code, either literally or in somewhat modified form. In some cases such repetitions may serve to clarify what is said, but often they simply amplify or emphasize a message" (1977, p. 16).

Por otro lado, a través de la inclusión de citas latinas el autor marca su propia opinión.

38. No hallo ninguno que mejor tenga tratada esta materia como Auicenna enla primera fen de su quarto. Pues pongamos la diffinicion de esta enfermedad segun su opinion. Son las viruelas/ o morbiles pustule parue per natura expulse in partibus cutaneis prouenientes ab ebullicione sanguinis: in qua fit separatio aliquarum partium \& remanet sanguis depuratus. Pues es necessario ver muchas cosas. (Libro de las comadres 1541, fol. 66v)

39. Puedesertambienalgunamaladisposicion causandodolorintenso/ypor serla substancia dela madre neruosa/ comuna ser a los miembros principales como dize Auicenna enla quinta fen del quarto ibi. Rerui propter vehementiam sensus \& eorum continuitatem cum cerebro accidit ex lesione ipsorum dolores valde magni \& lesiones sicut spasmus: per mixtio rationis \& multa. Y esto es cosa vera. (Libro de las comadres 1541, fol. 102v)

40. [...] de la qual dixo Auicena, Et scias quod medicina temperata curat omnes discrassias. Que es la mayor maxima y proposicion que esta escrita, [...] (Discurso y despertador preservativo 1594, fol. 12v)

Usualmente, el escritor se identifica con la autoridad citada, como se puede notar en el ejemplo 38 con el comentario que introduce la cita (No hallo ninguno que mejor tenga tratada esta materia como Auicenna), en 39, en donde se insiste en la veracidad de lo dicho 
( $Y$ esto es cosa vera) y en 40 con la valoración positiva del medicamento descrito por Avicena (Que es la mayor maxima y proposicion que esta escrita). También se dan pasajes en los cuales el autor cita a varias autoridades médicas y evalúa las distintas opiniones al compararlas, pero hay que tomar en cuenta que la ciencia en esta época, por los principios de la escolástica, se basa en la confianza absoluta por lo que dicen las autoridades (Siraisi, 1990, pp. 33 y 104).

\subsubsection{Organización textual}

El empleo de code-switching en textos escritos sirve para organizar el contenido y la estructura del manuscrito de las obras médicas. Por lo tanto, es común encontrar segmentos latinos al comienzo y al final de los manuscritos. Como se mencionó anteriormente, en algunos textos la dedicatoria completa está escrita en latín:

41. DOMINO DON ALfonso Gregorio, Caesaraugustano Archipraesuli dignissimo, ac catholici Regis nostri, consiliario integerrimo. Hieronymus Soriano Medicinae doctor. S.P.D. QVAM recte veteres Greci de rebus omnibus senserint,vel ex prouerbio AEisunir, vdaeisunir Praesul illustrissime quilibet conijcere poterit. Vnus etenim aiebant vir nullus est vir. [...] (Libro de experimentos médicos 1598, fol. 1r)

Las dedicatorias incluidas al inicio de las obras médicas se distinguen del contenido de la obra en general, la cual obviamente trata un tema propio del ámbito de la medicina. Además, también difieren en cuanto al público al que están dirigidos, pues las dedicatorias se escriben para los señores de la realeza que comisionaban la obra, mientras que el texto en sí estaba pensado ya fuera para el pueblo (como en el caso de los tratados de peste) ${ }^{37}$ o para los miembros de la comunidad científica. La composición latina, debido al prestigio de esta lengua, sirve para proyectar una imagen positiva del escritor, en tanto que demuestra conocimientos de latín y lo posiciona como parte de los médicos que se habían formado profesionalmente dentro de la tradición clásica y erudita. ${ }^{38}$

Por otro lado, el uso del latín en los prólogos y dedicatorias de los textos médicos evidentemente no tiene ninguna relevancia en cuanto al contenido de la materia medica, sino que cumple funciones decorativas, pues era usual comenzar las obras o los capítulos de las obras con fragmentos latinos, ${ }^{39}$ y es una forma de transmitir respeto hacia los señores que habían encargado la obra por comisión. ${ }^{40}$ Esto también se observa en el uso de fórmulas de agradecimiento (gratias) y de saludo (salve) agregadas al final de las dedicatorias, incluso cuando estas estaban redactadas en castellano. Tales fórmulas de agradecimientos, saludos y de alabanzas (como laus deo o solo deo) se utilizan convencionalmente para concluir las obras médicas. En este punto del texto es común que el escritor termine con un agradecimiento general o divino, como en el ejemplo 42, o que se dirija nuevamente a los lectores para insistir sobre la utilidad de su tratado, como en el ejemplo 43:

42. [...] assi este cuydado y trabajo ha de resultar en seruicio de la diuina Magestad, $y$ del Rey nuestro Señor, y bien vniuersal destos Reynos. LAVS DEO. (Defensa de las criaturas de tierna edad 1604, fol. 54v)

43. Es buena para el yjada \& para las vlceras del pulmon. Es prouocatiuo de sueño. Sana las vlceras intrinsicas. Es contra quien ha tomado algun veneno o ponçoña. Laus deo HAgo fin a este tractado intitulado el Morbo serpentino. Rogando a los lectores que tomen el mi buen zelo \& desseo que tuue de aprouechar a los que de lo en el contenido tuuieren necessidad. E si en algo soy digno de enmienda: por algun deffecto que en el aya: que lo 
enmienden, mirando mi buen desseo. Deo A Gloria de nuestro señor Jesu christo: \& de su gloriosa \& bendita madre \& señora nuestra acabose la presente obra en Seuilla. La qual se imprimio a costa del autor della. Por Andres de burgos vezino de Granada estante en Seuilla Impressor de libros a veynte \& ocho dias del mes de Nouiembre de mil \& quinientos y quarenta y dos años. Gratias (Contra el mal serpentino 1542, fol.81v, fol. 82r)

Estas expresiones también se emplean para cerrar una sección del texto y pasar a otra. Así, marcan la transición de una dedicatoria, por ejemplo, al contenido de la obra médica propiamente, o bien, señalan el final del texto para luego dar paso a la tabla de contenidos, lo cual se da en el texto del Manual contra la gota (ejemplo 44), en donde además se cierra con una frase latina religiosa. ${ }^{41}$ Igualmente, dentro de la materia medica pueden indicar un cambio de tema, como en el ejemplo 45, en el cual se pasa de hablar de las propiedades de los pescados como alimento a la enumeración de los beneficios del romero y sus recetas:

44. Porque desto se sigue la conseruacion del temperamento en su perfecto estado de salud: la qual si con la gracia del celestial y soberano Medico; con muy grande razon diremos desta medicina, Venerunt nobis omnia bona pariter cum illa. LAVS DEO. (Manual contra la gota 1589, fol. 107r, 106v)

45. Lo vno por la agudeza delas species \& lo otro por la grand vascosidad \& vmidad que se enello detiene delos pescados onde este pan es daño alos que lo vsan comer. otrosi dezimos que los pescados salados \& sal presos son mejores de comer cozidos que en otra manera. Deo gratias: Aqui comiençan las Recebtas del romero en que declara las grandes virtudes que tiene: \& otras reçebtas muy marauillosas. (Macer 1518, fol. 35r)

En relación con los cambios de tipo de texto, Pahta (2011, p. 128) indica que las recetas en textos de medicina del inglés moderno temprano suelen presentar elementos de codeswitching, lo cual marca el cambio de un tipo textual a otro. No obstante, en los textos castellanos estudiados esto solo sucede en el Libro de las comadres. ${ }^{42}$ De todas formas, se observan las mismas características que plantea Pahta, pues las medidas y los nombres de los ingredientes se escriben en latín, en griego o en árabe, mientras que las indicaciones están en español:

46. Recipe trociscorum de charabe/ trociscorum de terra figillata .anna onzas .ij. attanasie immortalis onza.j. media margaritarum coralis rubei anna. onza .j. cornu cerui vsti onza .j. media cum sirupo violato fiat admodum opiate. Del qual le daran dos vezes al dia con estas aguas. Recipe aquarum plantiginis violata \& endiuieanna dracmas iiij. misceantur. Despues hagan le este perfume. (Libro de las comadres 1541, fol. 94r)

Esto se debe a que las medidas y los ingredientes están de cierta manera estandarizados, pues las mismas recetas se encuentran en otras obras médicas contemporáneas o anteriores. Por lo tanto, se asume que esa información es manejada por el público, mientras que las indicaciones sobre el uso, la preparación y el consumo de las recetas, que se considera como la información más importante, se escriben en español.

Otro lugar en donde se utiliza el latín es en el margen del manuscrito. Esto ocurre en textos como el Libro del parto humano, el Tractado del uso de las mugeres y el Libro de la enfermedad de las bubas, entre otros. Como se mencionó más arriba, algunos escritores aprovechan este espacio para nombrar el título o el capítulo de una obra a la cual hacen referencia: ${ }^{43}$

47. Las señales que luego parecen especialmente quando el mal se pega por coito, o accesso carnal son las que enseña Fernelio, diziendo, que salen en las partes baxas y 
deshonestas, postillas, llagas malignas, materias por el caño, y encordios. AD. [Libro .6. de partium morbis capitulo .20. de lue venerea] (Libro de la enfermedad de las bubas 1600, fol. 14r)

A veces se incluye una cita junto con la referencia brindada en la adenda:

48. Dixe por la mayor parte, porque puede tambien el parto natural allegar al decimo mes, y al vndecimo como lo trae Aristoteles y Hippocrates, en diuersos lugares, Aristoteles en el .7. de historia Animalium, Hippocrates en el libro de Natura pueri, y en el libro de Septimestri partu, y el sapientissimo Rey en el libro de la sabiduria ansi. Sum quidem \& ego mortalis homo, similis omnibus ex genere terreno, \& illius qui prior factus est, \& in ventre matris fiugratus sum caro, decem mensium tempore coagulatus sum, in sanguine ex semine hominis, AD. Salomon y el excelentissimo poeta. Incipe parue puer(r) su cogn[o] scere matrem. Matri longa decem tulerunt fastidia menses. AD. Virgilio in bucolicis. (Libro del parto humano 1580, fol. 13v)

$\mathrm{O}$ bien, la nota al margen incluye el fragmento original latino que se ha citado en el cuerpo del texto, como en el siguiente ejemplo, en donde la adenda se encuentra en la dedicatoria de la obra:

49. A LA CATOLICA MAGESTAD DEL REY DON FELIPE SEGVNDO. SEÑOR. ENTRE Las verdades infalibles del Espiritu Santo, nos dize esta la Sabiduria. Que el altissimo Dios crio de la tierra l(e)[a] medicina. AD. Altissimus creauit de terra medicinam. Ecclesiastes capitulo .38. (Discurso de la gota 1594, fol. 2r)

50. pues las obras de Dios todas son AD. Dei perfecta sunt opera. Deuteronomium capitulo .32. perfectissimas, (Discurso de la gota 1594, fol. 2v, 3r)

Estas adiciones al margen de los manuscritos eran propias de la escritura de manuscritos de la época. Más allá de esto, cuando las notas incluyen información referencial e intertextual, funcionan como una guía para el lector, ya que le facilitan la lectura a través del manuscrito.

\section{El code-switching como práctica de escritura}

En este trabajo se han descrito las formas bajo las cuales se da la alternancia de códigos latín/español y se ha elaborado sobre las funciones discursivas que implica el uso del latín en textos principalmente escritos en español. Se ha planteado que los pasajes latinos abarcan desde palabras sueltas hasta secuencias textuales relativamente amplias y que su inclusión sirve para referirse a términos propios de la profesión médica, citar autores de la tradición erudita como refuerzo argumentativo y organizar tanto el contenido como la estructura del manuscrito.

Debido a que el empleo del latín tiene que ver con cómo se relacionan los autores de estos textos de medicina en lengua vernácula con los mecenas, los lectores laicos y los miembros de la comunidad científica, se sostiene que el code-switching forma parte de una práctica de escritura propia de este tipo de textos. Específicamente, los fragmentos latinos en las dedicatorias de las obras médicas, aparte de ser una especie de elemento decorativo, son una manera de transmitir respeto a los miembros de la nobleza y a los eclesiásticos que contrataban a los médicos y cirujanos, lo cual también se realiza a través de expresiones formulaicas de saludos y agradecimientos. Este carácter convencional, puesto que la escritura de prólogos y dedicatorias comúnmente se hacía en latín o en latín/castellano de forma paralela, surge de nuevo 
en la inclusión de expresiones metadiscursivas que enmarcan los textos dentro de una norma de escritura propia de un registro profesional. Con respecto a la relación de los escritores con los lectores laicos y con lectores de la comunidad médica que no necesariamente tenían conocimientos de latín, se observa que hay una conciencia lingüística por parte de los autores con respecto a su público meta. Esto se hace explícito mediante la confrontación de términos latinos con su equivalente en castellano y mediante la traducción o paráfrasis de citas y expresiones latinas.

Por último, debido a que la lengua latina era una señal de prestigio y de formación universitaria, su empleo como una práctica de intertextualidad al citar diversas obras y autores reconocidos es un recurso para marcar la pertenencia de grupo. De esta manera, los escritores se posicionan como profesionales expertos que tienen a su disposición todo un abanico de conocimiento compartido con los miembros de la comunidad científica, lo cual contribuye a forjar una imagen de médico instruido. Además, como el uso de citas da credibilidad y autoridad a los médicos escritores, estos pasajes cumplen la función retórica de reforzar sus propios argumentos y de persuadir a los lectores. La función persuasiva del code-swithing no solo se observa en pasajes argumentativos, sino también en textos instructivos en donde el propósito del médico es convencer al lector de que siga una instrucción.

\section{Notas}

1. Se trata de los trabajos clásicos de Poplack (1979) y Bloom y Gumperz (1972), ambos en Auer (1988).

2. Vid. la reseña completa en Sebba (2012, pp. 3-5).

3. Se trata del trabajo de Jonsson (2005), citado en Sebba (2012, p. 4).

4. También merece la pena mencionar las actas del congreso Il bilinguismo medico fra Tardoantico e Medioevo (Urso, 2012).

5. En un reciente estudio Montaner Frutos (2012, parr. 74) se refiere al cambio de código latín/romance como una estrategia conversacional en un documento de comienzos de la Edad Media ibérica.

6. A su vez, el progreso de la medicina se enmarca dentro del llamado Renacimiento del siglo XII: "In the rapid development of western European society that took place between about 1050 and 1225 and is often referred to as the "twelfth-century Renaissance," a population increase, economic growth, urbanization, the development of more sophisticated forms of secular and ecclesiastical government and administration, the growth of professional specialization and of occupations requiring literacy, the multiplication of schools, and the enlargement of philosophical, scientific, and technical learning were interwoven and interdependent phenomena. All had a marked impact on the study and the practice of medicine" (Siraisi, 1990, p. 13).

7. Vid. Sánchez González de Herrero y Vázquez de Benito (2010, p. 80) y Gutiérrez Rodilla (1998, p. 64).

8. No es un movimiento aislado, claro está, pues desde el siglo XIII con Alfonso X se emprende la tarea de redactar la historia de España y las obras de derecho en castellano (vid. Gutiérrez Rodilla, 1998, p. 64).

9. Vid. Sánchez González de Herrero y Vázquez de Benito (2010, p. 79).

10. El mundo de la medicina medieval está lleno de jerarquías: “[...] no estaban a la misma altura los cirujanos, autorizados por el Protomedicato o las Cofradías para desempeñar sus funciones, que los barberos y sangradores con una actividad más o menos reconocida o tolerada. Estos, por su parte, estaban por encima de los empíricos especializados en cometidos terapéuticos concretos, generalmente de tipo quirúrgico, tales como los algebristas, hernistas, litotomistas o comadres" (Gutiérrez Rodilla, 2009, p. 41). Según Siraisi (1990, p. 20), este tipo de jerarquía se daba en casi toda Europa occidental.

11. Estas discusiones se encuentran ya sea en los prólogos o en el cuerpo de las obras médicas (Santamaría Hernández, 2003, p. 731).

12. Se trata de más de 2500000 palabras (Gago Jover, Herrera y González de Fauve, 2015). 
13. Vid. la discusión de Crespo y Moskowich (2006, pp. 51-53) sobre la dificultad de establecer criterios claros para diferenciar el code-switching de los préstamos léxicos. En términos generales, el préstamo lingüístico involucra el uso de vocablos de origen extranjero que ya han sido integrados en la otra lengua, por lo cual se supone que el code-switching precede a los préstamos (ibid., p. 51). Además, mientras que la alternancia de código requiere de una cierta competencia de los idiomas empleados, el uso de préstamos puede darse por parte de hablantes monolingües sin estar conscientes que se trata de elementos léxicos extranjeros (ibid., p. 52). En todo caso, ambos fenómenos ocurren a través del contacto lingüístico, por lo cual evidentemente son hechos sociales.

14. Estas dos últimas formas se utilizan al inicio de cláusula dentro de un texto (Lleal, 2017).

15. Vid. Niiranen (2011, p. 65).

16. Vid. Gil-Sotres (1994, p. 136).

17. Niiranen $(2011$, p. 74) observa lo mismo con respecto a las prácticas de citación en textos médicos en inglés antiguo, en donde no se da la preocupación por referenciar correctamente, de acuerdo con patrones contemporáneos.

18. Los paréntesis representan la puntuación.

19. Hay que recordar que los escritores de medicina encontraban su fuente económica, necesaria para la publicación de sus obras, en miembros de la nobleza o del clero (Gutiérrez Rodilla, 2009, p. 40).

20. Además de las funciones de intertextualidad, terminología y organización textual, Pahta (2011) señala el uso del code-switching en las recetas médicas incluidas en textos médicos del inglés moderno temprano como una categoría aparte.

21. La literatura médica en lenguas vernáculas está basada en fuentes árabes y griegas, escritas en su mayor parte en latín (Sánchez González de Herrero y Vázquez de Benito, 2010, p. 79).

22. Otras lenguas utilizadas como alternancia de código son el árabe y otras lenguas romances, como el italiano, utilizado en la narración de esta anécdota: "[...] llego a el vn Pelegrino con vn Banastillo de Rosarios de Euano que alla nombran Coronas para que su Santidad las bendixese: pues como el Pelegrino se arrodillase al passo, y alçasse el Banastillo, hasta que los Rosarios encontrasen con los dedos del Pontifice, y los sintiese frios, dio vn grande grito, diziendo que cosa e questa? y le respondiessen, Pater Sancte Coronas para bendezir, dixo el Pontifice lastimado del toque, maledette siano le Corone; como le replicasse por la bendicion; orfu, respondio, siano benedette." (Manual contra la gota 1589, fol. 20r, 20v)

23. Vid. Sebastián Yarza (1964, p. 1211).

24. Cfr. Lleal (2017).

25. Como ya se ha mencionado, los miembros de la comunidad científica que dominan el latín son aquellos que se han formado en la universidad, quienes a su vez ocupan un lugar alto en la jerarquía de médicos entre los siglos XIII y XVII (Siraisi, 1990, p. 48).

26. Vid. Segura Munguía (2003, p. 335).

27. Vid. Pérez Pascual (1992, p. 755).

28. Vid. Lleal (2017).

29. En realidad el término castellano es tomado del occitano antiguo (Lleal, 2017). Se trata de una "(p)lanta compuesta, de flores blancas con estambres amarillos y raíz de sabor picante usada en medicina".

30. Este proceso, que tiene que ver con la formación de los lenguajes de especialidad en las lenguas vernáculas, inició en España a mediados del siglo XIII en el taller y cancillería alfonsíes con las obras de traducción, lo cual significó un aumento inmenso del léxico y las posibilidades de expresión en castellano (Clavería Nadal, 2004, p. 72).

31. En los textos más recientes también se citan obras relativamente contemporáneas. Por ejemplo, en el Tratado de raras y peregrinas yervas se cita a un médico de principios del siglo XVI conocido como Leonardo Fuchs, oriundo de Tubinga, Alemania, que había escrito un libro sobre hierbas y plantas medicinales que luego había sido traducido al español (cfr. Pardo Tomás, 1991): Leonardo Fusio en su Libro de su historia plantarum, en el capitulo .2. dize: que el Abrotano lo llama el vulgo cypres syluestre, por razon de la similitud, y olor que tiene con el; y en lengua Francesa lo llaman Abrotano, que es sarmentoso [...] (Tratado de raras y peregrinas yervas 1674, fol. 22v). 
32. El dominio del latín era un marcador de prestigio intelectual y social, pues quienes conocían esta lengua eran los médicos que habían recibido indudablemente una educación universitaria (Siraisi, 1990, p. 20).

33. Vid. Pahta (2011, p. 127), quien observa el mismo fenómeno en textos médicos del inglés moderno temprano.

34. Este es un texto traducido del latín (Practica seu Lilium medicinae, de 1303) al castellano, por lo cual más bien el traductor deja las citas en su idioma original en lugar de traducir al español.

35. El término 'máxima moral' es de Blum-Kulka, House y Kasper (1989, p. 288). Según las autoras, los hablantes recurren a las máximas morales como movimientos de apoyo para darle mayor credibilidad a sus solicitudes. Cf. Cruz Volio (2017) sobre el empleo de máximas morales para modular actos de habla directivos en español medieval.

36. Además de que aparecen en otros tratados médicos de la época (vid. Nota 16). La cita del ejemplo 35 es parte del Flos medicinae Scholae Salerni.

37. De acuerdo con Gutiérrez Rodilla (2009, p. 40), los tratados de peste y otros tipos de regímenes sanitarios se escribían en principio para los nobles y para las autoridades civiles y eclesiásticas con el fin de que impusieran sus órdenes sanitarias sobre el resto de la población.

38. De acuerdo con Siraisi (1990, pp. 35 y 36), los médicos y cirujanos graduados consideraban que sus conocimientos de medicina eran bastante más superiores y mejor valorados socialmente que los de los practicantes empíricos, lo cual se evidencia en el uso de términos despectivos para referirse a estos, como "iletrados", "rústicos" y "viejas", entre otros. El prestigio intelectual y la fama de que gozaban los médicos de formación universitaria se mantuvo desde el siglo XIV hasta bien entrado el Renacimiento (ibid., p. 43).

39. Vid. Pahta: “[...] foreign-language segments also occur in communicative functions connected more generally with the culture of knowledge and writing and literacy practices of the time. In some of them, as in the opening example of the chapter, the change of language seems more motivated by decorative or image-creating purposes than by a content-related communicative function" (2011, p. 123).

40. Debe tenerse en cuenta que los miembros de la nobleza, así como eclesiásticos de alto rango, estaban en una posición de superioridad y tenían el poder de contratar médicos y cirujanos como sus médicos personales (Siraisi, 1990, p. 36).

41. La frase venerunt mihi omnia bona pariter cum illa es atribuida a Alberto Magno (ca. 1195-1280), en: Albertus Magnus, Opera Omnia (Editio Parisiensis), ed. A. et Ae. Borgnet, Paris 1890-99, vol. 37.

42. Según Pahta (2011, p. 129), las recetas en latín ocurren con mayor frecuencia en obras relacionadas con la comunidad profesional, como textos de cirugía y otros textos académicos dirigidos a médicos universitarios.

43. En las ediciones de la Digital Library of Old Spanish Texts esto se indica mediante la abreviatura "AD" (adenda).

\section{Bibliografía}

\section{Fuentes primarias}

Bau, A. M. (Ed.). (2012). Defensa de las criaturas de tierna edad, y algunas dudas y advertencias cerca de la curación y conservación de la salud. Spanish Medical Texts. Digital Library of Old Spanish Texts. Digital Library of Old Spanish Texts. Hispanic Seminary of Medieval Studies. Recuperado de http://www.hispanicseminary.org/t\&c/ med/index-en.htm

Bau, A. M. (Ed.). (2012). Discurso particular preservativo de la gota. Spanish Medical Texts. Digital Library of Old Spanish Texts. Digital Library of Old Spanish Texts. Hispanic Seminary of Medieval Studies. Recuperado de http://www.hispanicseminary.org/t\&c/ med/index-en.htm 
Bau, A. M. (Ed.). (2012). Discurso y despertador preservativo de corrimientos y enfermedades. Spanish Medical Texts. Digital Library of Old Spanish Texts. Digital Library of Old Spanish Texts. Hispanic Seminary of Medieval Studies. Recuperado de http://www. hispanicseminary.org/t\&c/med/index-en.htm

Bau, A. M. (Ed.). (2012). Libro que trata de la enfermedad de las bubas. Spanish Medical Texts. Digital Library of Old Spanish Texts. Digital Library of Old Spanish Texts. Hispanic Seminary of Medieval Studies. Recuperado de http://www.hispanicseminary.org/t\&c/ med/index-en.htm

Bau, A. M. (Ed.). (2012). Tratado de raras y peregrinas yervas, que se han hallado en esta Corte. Spanish Medical Texts. Digital Library of Old Spanish Texts. Digital Library of Old Spanish Texts. Hispanic Seminary of Medieval Studies. Recuperado de http://www. hispanicseminary.org/t\&c/med/index-en.htm

Campagne, F. A. y Bau, A. M. (Eds.). (2012). Libro intitulado del parto humano. Spanish Medical Texts. Digital Library of Old Spanish Texts. Digital Library of Old Spanish Texts. Hispanic Seminary of Medieval Studies. Recuperado de http://www. hispanicseminary.org/t\&c/med/index-en.htm

Canavese, G. (Ed.). (2012). Tractado del vso de las mugeres. Spanish Medical Texts. Digital Library of Old Spanish Texts. Digital Library of Old Spanish Texts. Hispanic Seminary of Medieval Studies. Recuperado de http://www.hispanicseminary.org/t\&c/ med/index-en.htm

Capuano, T. M. (Ed.). (2012). Libro de medecina llamado macer. Spanish Medical Texts. Digital Library of Old Spanish Texts. Digital Library of Old Spanish Texts. Hispanic Seminary of Medieval Studies. Recuperado de http://www.hispanicseminary.org/t\&c/ med/index-en.htm

Conerly, P., Ardemagni, E. J. y Richards, R. M. (Eds.). (2012). Macer herbolario. Spanish Medical Texts. Digital Library of Old Spanish Texts. Digital Library of Old Spanish Texts. Hispanic Seminary of Medieval Studies. Recuperado de http://www. hispanicseminary.org/t\&c/med/index-en.htm

Cull, J. y Wasick, C. (Eds.). (2012). Lilio de medicina. Spanish Medical Texts. Digital Library of Old Spanish Texts. Digital Library of Old Spanish Texts. Hispanic Seminary of Medieval Studies. Recuperado de http://www.hispanicseminary.org/t\&c/med/index-en.htm

de Forteza, P. (Ed.). (2012). Libro de experimentos médicos, fáciles y verdaderos, recopilados de grauíssimos autores. Spanish Medical Texts. Digital Library of Old Spanish Texts. Digital Library of Old Spanish Texts. Hispanic Seminary of Medieval Studies. Recuperado de http://www.hispanicseminary.org/t\&c/med/index-en.htm

de Forteza, P. (Ed.). (2012). Tratado breve y compendioso sobre la maravillosa obra de la boca y dentadura. Spanish Medical Texts. Digital Library of Old Spanish Texts. Digital Library of Old Spanish Texts. Hispanic Seminary of Medieval Studies. Recuperado de http://www.hispanicseminary.org/t\&c/med/index-en.htm

Espina, A. (Ed.). (2012). Tratado contra toda pestilencia \& ayre corrupto. Spanish Medical Texts. Digital Library of Old Spanish Texts. Digital Library of Old Spanish Texts. Hispanic Seminary of Medieval Studies. Recuperado de http://www.hispanicseminary. org/t\&c/med/index-en.htm 
Fernández, S. (Ed.). (2012). De las melecinas. Spanish Medical Texts. Digital Library of Old Spanish Texts. Digital Library of Old Spanish Texts. Hispanic Seminary of Medieval Studies. Recuperado de http://www.hispanicseminary.org/t\&c/med/index-en.htm

Gubitosi, P. (Ed.). (2012). Tractado llamado fructo de todos los auctos: contra el mal serpentino. Spanish Medical Texts. Digital Library of Old Spanish Texts. Digital Library of Old Spanish Texts. Hispanic Seminary of Medieval Studies. Recuperado de http://www. hispanicseminary.org/t\&c/med/index-en.htm

Herrera, M. T. (Ed.). (2012). Tratado de cirugía. Spanish Medical Texts. Digital Library of Old Spanish Texts. Digital Library of Old Spanish Texts. Hispanic Seminary of Medieval Studies. Recuperado de http://www.hispanicseminary.org/t\&c/med/index-en.htm

Incarnato, C. E. (Ed.). (2012). Enchiridion o Manual instrumento de salud contra el morbo articular que llaman gota. Spanish Medical Texts. Digital Library of Old Spanish Texts. Digital Library of Old Spanish Texts. Hispanic Seminary of Medieval Studies. Recuperado de http://www.hispanicseminary.org/t\&c/med/index-en.htm

Kaste, L., Nitti, J. y Jonxis-Henkemans, W. (Eds.). (2012). Lapidarios de Alfonso X. Spanish Medical Texts. Digital Library of Old Spanish Texts. Digital Library of Old Spanish Texts. Hispanic Seminary of Medieval Studies. Recuperado de http://www. hispanicseminary.org/t\&c/med/index-en.htm

Piñeyrua, A. (Ed.). (2012). Libro del arte de las comadres o madrinas y del regimiento de las preñadas y paridas y de los niños. Spanish Medical Texts. Digital Library of Old Spanish Texts. Digital Library of Old Spanish Texts. Hispanic Seminary of Medieval Studies. Recuperado de http://www.hispanicseminary.org/t\&c/med/index-en.htm

Richards, R.M. (Ed.). (2012). Tratado de las fiebres. Spanish Medical Texts. Digital Library of Old Spanish Texts. Digital Library of Old Spanish Texts. Hispanic Seminary of Medieval Studies. Recuperado de http://www.hispanicseminary.org/t\&c/med/index-en.htm

\section{Referencias}

Auer, P. (1988). Introduction: Bilingual Conversation revisited. En P. Auer (Ed.). CodeSwitching in Conversation: Language, Interaction and Identity. (pp. 1-24). London: Routledge.

Blum-Kulka, S., House, J. y Kasper, G. (Eds.). (1989). Cross-cultural Pragmatics: Requests and Apologies. (Vol. Advances in Discourse Processes). Norwood NJ: Ablex.

Clavería Nadal, G. (2004). Aproximación a los inicios de la lengua de la medicina: La terminología patológica en la obra Alfonsí . Revista de Investigación Lingüística. 7, 71-90.

Crespo, B., y Moskowich, I. (2006). Latin forms in vernacular scientific writing: Codeswitching or borrowing? En R. W. McConchie, O. Timofeeva, H. Tissari y T. Säily (Eds.). Selected proceedings of the 2005 Symposium on New Approaches in English Historical Lexis (HEL-LEX). (pp. 51-59). Somerville, MA: Cascadilla Press.

Cruz Volio, G. (2017). Actos de habla y modulación discursiva en español medieval: Representaciones de (des)cortesía verbal histórica. (Vols. Studia Romanica et Linguistica, 50). New York: Peter Lang. 
Davidson, M. C. (2005). Discourse features of code-switching in legal reports in late medieval England. En J. Skaffari, M. Peikola, R. Carroll, R. Hiltunen y B. Wårvik (Eds.). Opening Windows on Texts and Discourses of the Past. (Vol. Pragmatics \& Beyond New Series, pp. 343-352). Amsterdam/Philadelphia: John Benjamins.

Fløttum, K., Dahl, T. y Kinn, T. (2006). Academic Voices: Across Languages and Disciplines. Amsterdam/Philadelphia: John Benjamins.

Gago Jover, F., Herrera, M. T. y González de Fauve, M. E. (2015). HSMS : Digital Library of Old Spanish Texts. Recuperado de http://www.hispanicseminary.org/t\&c/med/index-en.htm

Gil-Sotres, P. (1994). Derivation and revulsion: the theory and practice of medieval phlebotomy. En L. García-Ballester, R. French, J. Arrizabalaga, y A. Cunningham (Eds.). Practical Medicine from Salerno to the Black Death. (pp. 110-157). Cambridge: Cambridge University Press.

Gumperz, J. J. (1977). The sociolinguistic significance of conversational code-switching. RELC Journal. 8 (2), 1-34.

Gutiérrez Rodilla, B. (1998). La ciencia empieza en la palabra. Análisis e historia del lenguaje científico. Barcelona: Ediciones Península.

Gutiérrez Rodilla, B. M. (2009). La adecuación lingüística al destinatario en los textos médicos instructivos y de divulgación del renacimiento castellano. Res Diachronicae. 7, 37-46.

Heller, M. (Ed.). (1988). Codeswitching: Anthropological and Sociolinguistic Perspectives. (Vols. Contributions to the Sociology of Language, 48). Berlin/New York/Amsterdam: Mouton de Gruyter.

Hyland, K. (2005). Metadiscourse: Exploring Interaction in Writing. London/New York: Continuum.

Lleal, C. (Ed.). (10 de 02 de 2017). DiCCA XV - Diccionario del castellano del siglo XV en la Corona de Aragón. Recuperado de http://ghcl.ub.edu/diccaxv/pages/presentation

Montaner Frutos, A. (13 de 06 de 2012). El continuo diastrático de la espectroglosia latinorromance ibérica medieval .e-Spania. Recuperado de http://journals.openedition. org/e-spania/21093?lang=en

Myers-Scotton, C. (1993). Duelling Languages: Grammatical Structure in Codeswitching. New York: Clarendon Press.

Niiranen, S. (2011). The Authority of words: The healing power of vernacular, Latin and other languages in an Occitan remedy collection. Mirator. 12, 54-75.

Nurmi, A. y Pahta, P. (2012). Multilingual Practices in Women's English Correspondence 1400-1800. Language Mixing and Code-Switching in Writing Approaches to MixedLanguage Written Discourse. (pp. 44-67). New York/London: Routledge.

Pahta, P. (2011). Code-switching in Early Modern English medical writing. En I. Taavitsainen y P. Pahta (Eds.). Medical Writing in Early Modern English. (pp. 115-134). Cambridge: Cambridge University Press.

Pardo Tomás, J. (1991). Ciencia y censura. La inquisición española y los libros científicos en los siglos XVI y XVII. Madrid: Consejo Superior de Investigaciones Científicas. 
Pérez Pascual, J. I. (1992). Algunas aportaciones de la SUMA DE LA FLOR DE CIRUGÍA. En Bartol Hernández, José Antonio, J. de Santiago Guervós y J. García Santos (Eds.). Estudios filológicos en homenaje a Eugenio de Bustos Tovar. (Vol. 2, pp. 749-760). Salamanca: Ediciones Universidad de Salamanca.

Sánchez González de Herrero, M. y Vázquez de Benito, M. (2010). La traducción de textos médicos medievales. Cuestiones en torno al léxico. Cuadernos del Instituto de Historia de la Lengua. 4, 77-103.

Santamaría Hernández, M. T. (1999). El léxico latino de la fisiología en los textos del humanismo médico valenciano: la tradición de spiritus. Myrtia: Revista de filología clásica. 14, 119-142.

Santamaría Hernández, M. T. (2003). La valoración de la lengua en los escritos del humanismo médico valenciano: latín, griego y vulgar. En F. G. Codina (Ed.). La Universitat de València i l' Humanime: Studia Humanitatis y renovació cultural a Europa i el Nou Món (Valencia, Abril 2000). (pp. 713-740). Valencia: Universidad.

Schendl, H. (2012). Literacy, Multilingualism and Code-switching in Early English Written Texts. En M. Sebba, S. Mahootian y C. Jonsson (Eds.). Language Mixing and CodeSwitching in Writing. (pp. 44-67). New York/London: Routledge.

Diccionario griego-español. (1964). (F. I. Sebastián Yarza, tr.) Barcelona: Sopena.

Sebba, M. (2012). Researching and Theorising Multilingual Texts. En M. Sebba, S. Mahootian y C. Jonsson (Eds.). Language Mixing and Code-Switching in Writing: Approaches to Mixed-Language Written Discourse. (pp. 1-26). New York/London: Routledge.

Segura Munguía, S. (2003). Nuevo diccionario etimológico Latín-Español y de las voces derivadas. Bilbao: Universidad de Deusto.

Siraisi, N. (1990). Medieval and Early Renaissance Medicine: An Introduction to Knowledge and Practice. Chicago: University of Chicago Press.

Thomas, J. A. y Sayahi, L. (2012). A Quantitative Analysis of Code-switching in the ArabicRomance Kharjas. Journal of Language Contact. 5 (2), 262-278.

Urso, A. M. (Ed.). (2012). Il bilinguismo medico fra Tardoantico e Medioevo. Università degli Studi di Messina.

Wei, L. (2000). Dimensions of bilingualism. En L. Wei (Ed.). The Bilingualismo Reader. (pp. 1-21). London/New York: Routledge. 